\title{
Fast-neutron radiation effects in a silica-core optical fiber studied by a CCD-camera spectrometer
}

\author{
D. L. Griscom, M. E. Gingerich, E. J. Friebele, M. Putnam, and W. Unruh
}

\begin{abstract}
A simple CCD-camera spectrometer was deployed at the Los Alamos Spallation Radiation Effects Facility to characterize fast-neutron irradiation effects in several silica-based optical fibers over the wavelength range $\sim 450-1100 \mathrm{~nm}$. The experimental arrangement allowed optical loss spectra to be developed from remotely recovered frame grabs at various times during irradiation without it being necessary to resort to cutback methods. Data recorded for a pure-silica-core/F-doped-silica-clad fiber displayed a peculiar artifact, which is described and mathematically modeled in terms of leaky modes propagating in an optical cladding that is substantially less susceptible to radiation-induced optical attenuation than is the core. Evidence from optical time-domain reflectometry supports the postulate that mode leakage into the cladding may be a result of light scattering from the tracks of ions displaced by the $14-\mathrm{MeV}$ neutrons. These results suggest that fibers with fluorine doping in the core, as well as in the cladding, would be relatively resistant to radiation-induced attenuation in the UV-visible spectral region.

Key words: Fiber optics, radiation effects, fast-neutron damage, CCD-camera spectrophotometer.
\end{abstract}

\section{Introduction}

Fusion power reactors based on magnetically confined plasmas depend critically on a wide variety of diagnostic systems, including optical systems operating in the 300-1500-nm wavelength range. ${ }^{1}$ In present-generation tokamaks, fiber optics are used to convey these light signals from ports near the reactor first wall to shielded instrument areas several tens of meters distant. In advanced deuterium-tritiumburning systems, these fibers must continue to operate under intense fluxes and fluences of high-energy neutrons (up to $14 \mathrm{MeV}$ ) and $\gamma$ rays. To observe the effects of high-energy radiations characteristic of the deuterium-tritium fusion reactor environment on candidate fiber waveguides, an experiment was designed for quick, low-cost deployment at the Los Alamos Spallation Radiation Effects Facility (LASREF) associated with the Los Alamos Meson Physics Facility (LAMPF). LASREF delivers a neu-

D. L. Griscom, M. E. Gingerich, and E. J. Friebele are with the Optical Sciences Division, Naval Research Laboratory, Washington, D.C. 20375. M. Putnam is with the Virginia Polytechnic Institute, Blacksburg, Virginia 24061. W. Unruh is with the Los Alamos National Laboratory, Los Alamos, New Mexico 87545.

Received 26 February 1993; revision received 2 September 1993. 0003-6935/94/061022-07\$06.00/0.

() 1994 Optical Society of America. tron spectrum reasonably modeling that of a deuterium-tritium fusion reactor. ${ }^{1}$ This paper briefly describes the experiment and presents highly instructive preliminary results obtained during the AugustSeptember 1992 operation of LAMPF.

\section{Experiment}

The experimental arrangement, shown in Fig. 1, consisted of using a lead-glass prism to disperse simultaneously the light emanating from several fibers on the focal plane of a charge-coupled-device (CCD) camera, so that the recorded images would consist of a stack of parallel streaks representing for each fiber the light intensity (convoluted with the camera sensitivity) versus the tangent of the angle of refraction. Two types of fibers were selected for the experiment: pure-silica-core/F-doped-silica-clad fibers from three separate manufacturers and one graded-index Ge-doped-silica-core fiber. All these fibers were multimode, with core diameters of 100 or $110 \mu \mathrm{m}$ and cladding diameters ranging from 120 to $140 \mu \mathrm{m}$; all the fibers had protective polymer coatings. Two lengths ( 1 and $20 \mathrm{~m}$ ) of each of these fibers were coiled on a single spool for insertion into a LASREF beam position delivering $7.5 \times 10^{10} \mathrm{n} / \mathrm{cm}^{2}$ at an average temperature of $\sim 30^{\circ} \mathrm{C}$. The two pigtail extensions of each of the eight fiber coils were led up through $\sim 5 \mathrm{~m}$ of radiation shielding, where they 


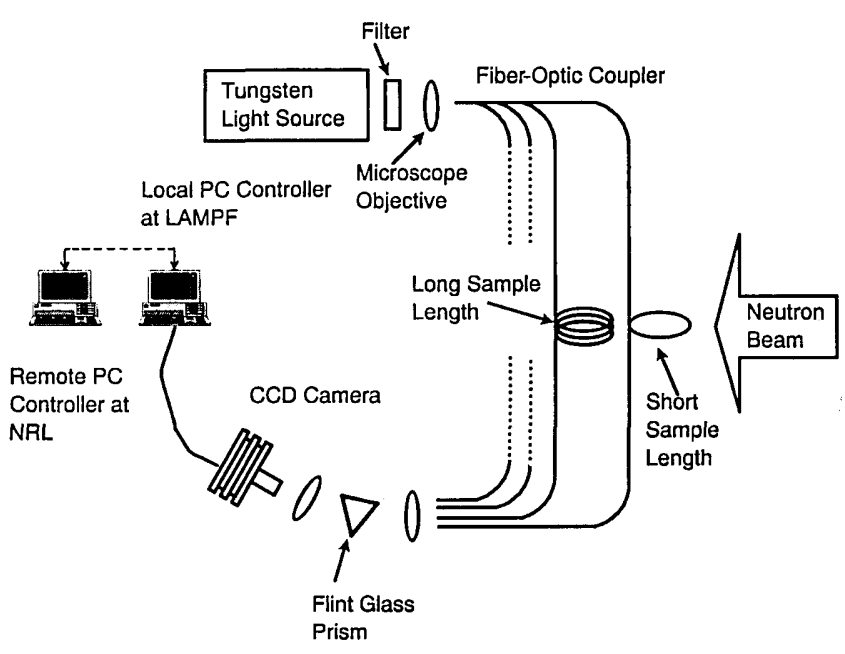

Fig. 1. Schematic diagram of experimental setup.

were fusion spliced to lead-in and lead-out runs of the identical fiber types leading from the top of the hole to the instrumentation area $\sim 40 \mathrm{~m}$ distant. The eight fiber lead ins were spliced to a pair of $1 \times 4$ fiber-optic couplers, which functioned as four-way beam splitters. Light intensities $\sim 1 \mu \mathrm{W}$ from a tungsten source were injected into the input pigtails of the two $1 \times 4$ couplers by the use of a microscope objective. Both long and short lengths of each experimental fiber type were spliced to the same coupler, so that (within the 0.4- $\mathrm{dB}$ precision of the coupler) the same light intensity was injected into the $20-\mathrm{m}$ sample length as was injected into the 1-m reference length. The cleaved spectrometer ends of the eight lead-out fibers were aligned in a vertically stacked array by means of a parallel set of $\mathrm{V}$ grooves in a metal block. The arrangement was such that the exit end of the array can be imagined as replacing the entrance slit in a conventional spectrometer. Each of the prismdispersed light streaks was individually calibrated in wavelength by inserting a series of eight sharp bandpass filters (ranging from 450 to $1100 \mathrm{~nm}$ ) between the light source and the microscope objective. Calibrations of wavelength versus horizontal pixel number were developed by fitting the recorded spot positions with a fifth-order polynomial.

The induced loss, $L$ (decibels per kilometer), in an effective sample length of $19 \mathrm{~m}(20 \mathrm{~m}-1 \mathrm{~m})$ of a given fiber type was then calculated as

$$
L(\lambda)=\frac{10}{0.019} \log _{10}\left[\frac{I_{0}(\lambda)}{I(\lambda)}\right]
$$

where $I(\lambda)$ and $I_{0}(\lambda)$ are the pixel intensities (with backgrounds subtracted) corresponding to wavelength $\lambda$ as measured for the $20-\mathrm{m}$ and $1-\mathrm{m}$ coils, respectively.

The idea of irradiating the short reference coil as well as the long sample coil was motivated by the desire to prevent the light transmitted through the long and short members of each fiber pair from ever differing by more than $13 \mathrm{~dB}$ in a given wavelength interval. The purpose of this constraint is to allow both transmission spectra to be recorded simultaneously in a single-frame grab with good signal to noise for the sample coil while averting saturation of the CCD-camera by the light transmitted by the reference length. As both fibers darken, data of equal quality can be obtained by increasing the integration time from as short as $0.01 \mathrm{~s}$ to as long as several hours, if needed. A second advantage of this arrangement is that the optical attenuation in the lead-in and lead-out fibers is automatically canceled in the process of calculating the optical loss, $L(\lambda)$, as described above. This cancellation is important because the up and down pigtails are comparable in length to the sample coil but, unlike the sample coil, are unavoidably exposed to a neutron flux that varies as a function of height above the beam centerline-a situation that would otherwise seriously complicate dosimetry. Coincidentally suppressed in the same process are the strong $\mathrm{OH}$ overtone absorption bands in the 40-m fiber lengths leading to and from the instrument area.

\section{Results}

It was expected that the transmitted light intensities of all eight fibers would be roughly the same before irradiation. However, it was found that the fiber with the thinnest cladding ( $7.5 \mu \mathrm{m}$, vis-à-vis 10 or 20 $\mu \mathrm{m}$ for the others) transmitted too little of the injected light to be measured. (Use of longer integration times was precluded by the blooming of adjacent streaks of the more highly transmitting fibers.) The poor transmission of this fiber was traced to light tunneling through the relatively thin cladding into the UV-cured epoxy used to reinforce the splices. Derived losses for the second of the pure-silica-core fibers were problematic; this was evidently due to a lossy splice on the reference fiber; for that reason, these data are not discussed further.

The reference length of the Ge-doped fiber broke shortly after the beginning of the experiment and could not be respliced. Nevertheless, the initially obtained data were sufficient to show induced losses above $\sim 750 \mathrm{~nm}$, which were considerably greater than losses in the pure-silica-core fibers - as expected based on earlier $\gamma$-ray and reactor irradiation studies. $^{2-8}$ Below $\sim 750 \mathrm{~nm}$, however, the apparent induced losses in the Ge-doped fiber dropped to unexpectedly low values. These data are not discussed further, except to remark that the anomalous results at shorter wavelengths are almost certain to have the same explanation as a similar effect observed for the third pure-silica-core/F-doped-silica-clad fiber of this study. The results for this last fiber, obtained from CeramOptec (core/cladding diameters, 100/120 $\mu \mathrm{m}$; core $\mathrm{OH}$ content, 40 parts in $10^{6}$ ) are presented below.

Figure 2 shows the transmitted light spectra $I(\lambda)$ for the sample and $I_{0}(\lambda)$ for the reference lengths of the CeramOptec fiber at an early stage of irradiation (fluence $=5 \times 10^{15} \mathrm{n} / \mathrm{cm}^{2}$ ). ${ }^{9} \quad$ These spectra comprise the intensity profile of the tungsten lamp source 


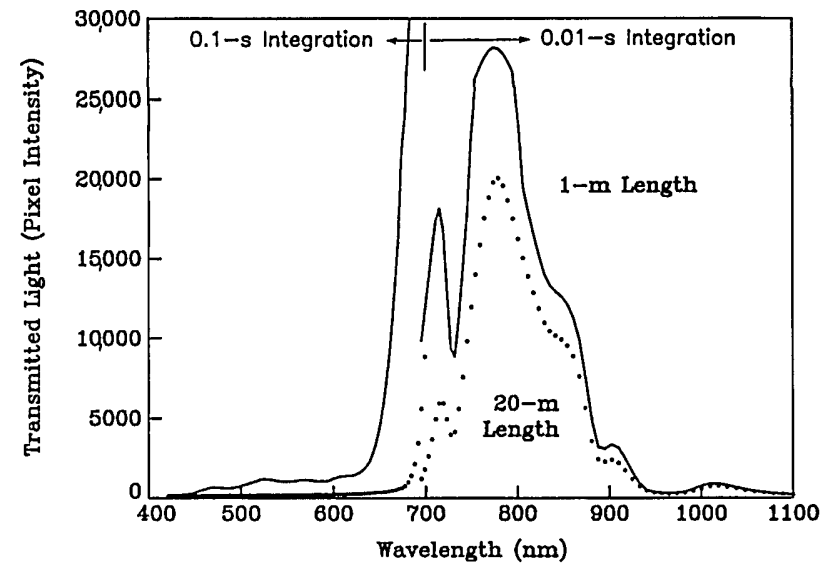

Fig. 2. Transmission spectra of CeramOptec fibers after accumulated neutron fluence ${ }^{9}$ of $5.1 \times 10^{15} \mathrm{n} / \mathrm{cm}^{2}$. Spectra below $700 \mathrm{~nm}$ are expanded by using a longer integration time.

multiplied by the camera sensitivity, as modified by absorption in the respective lengths of fiber. Below $\sim 700 \mathrm{~nm}$, where the beam-induced absorption had already become significant, a longer integration time was used to improve the signal-to-noise ratio. At the 0.1-s integration time, the background measured with the light source blocked was found to be essentially flat, showing no measurable streaks resulting from either room light or beam-induced luminescenses in the fibers (background pickup could be recorded for integration times $>100 \mathrm{~s}$, however).

Figure 3 illustrates the induced loss in the CeramOptec fiber calculated by means of Eq. (1) from transmission spectra recorded at various times during the beam-on period. The curves are labeled according to the accumulated fluence ${ }^{9}$ in units of $10^{14}$ $n / \mathrm{cm}^{2}$. Note that because of the cancellation of the attenuations in the long lead-in and lead-out fibers, the induced attenuation spectra of Fig. 3 exhibit only faint replications of the prominent first and second $\mathrm{OH}$ overtone bands near 950 and $725 \mathrm{~nm},{ }^{10}$ which are strongly apparent in the transmission spectra of Fig. 2.

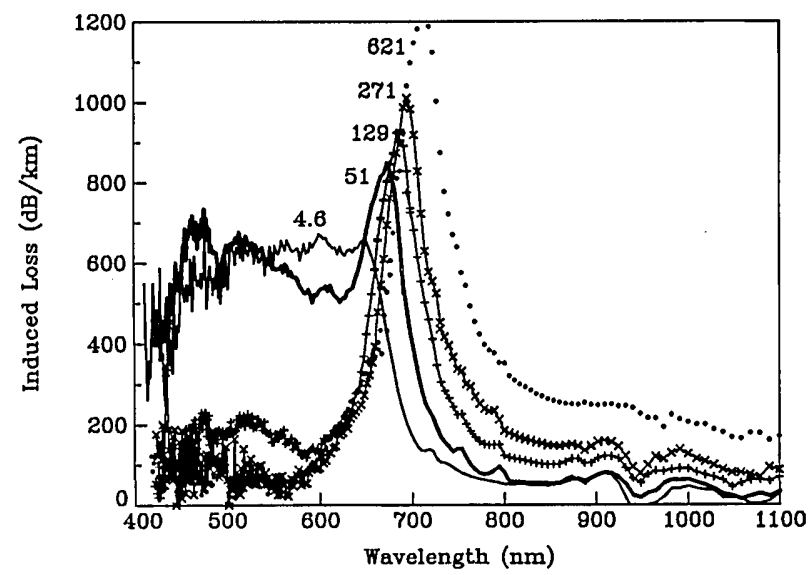

Fig. 3. Induced optical loss spectra of CeramOptec fiber during neutron irradiation. Numbers on the curves correspond to the neutron fluence ${ }^{9}$ (in units of $10^{14} \mathrm{n} / \mathrm{cm}^{2}$ ).
The induced absorption spectra of Fig. 3 are qualitatively reminiscent of spectra previously recorded for fibers and bulk silica samples exposed to equivalent doses of $\gamma$ rays. ${ }^{11-13}$ However, on closer inspection there are some crucial dissimilarities requiring explanation: First, the peaks near $700 \mathrm{~nm}$ in Fig. 3 are considerably sharper than the well-known bands peaking near 600-630 nm that are observed under $\gamma$ irradiation (e.g., Fig. 4). Second, contrary to previous observation, the currently observed peaks are increasingly red shifted with increasing neutron fluence above $\sim 10^{15} \mathrm{n} / \mathrm{cm}^{2}$. Third, the plateau seen in Fig. 3 in the wavelength regime between $\sim 400$ and $\sim 650 \mathrm{~nm}$ initially grows but then bleaches, as a function of increasing fluence. At the highest fluence, the induced loss in the 400-600-nm region falls back below $100 \mathrm{~dB} / \mathrm{km}$. Such radiation bleaching in high-purity fused silica in this spectral region is unprecedented and, in fact, cannot be explained except as an artifact. Nevertheless, as described below, the low induced loss measured in the visible region is a real effect, and an understanding of the present artifact gives a strong clue as to how a reasonably rad-hard fiber for fusion diagnostics might be designed.

\section{Mathematical Modeling}

Most optical fibers consist of a core (or light-guiding region) that conducts the bulk of the transmitted light, an optical cladding comprising a transparent material having a lower index of refraction, and a protective jacket (for detailed description of fiberoptic waveguides and telecommunications systems, see Refs. 14 and 15). If care is taken to inject light only into the fiber core, and the fiber is not bent or coiled to a radius below a few inches, only an evanescent wave penetrates the optical cladding. However, if such care is not taken and the protective jacket of the fiber happens to have an index of refraction lower than that of the optical cladding and is not too lossy, it is possible for light to propagate separately in the cladding. In many situations such cladding modes

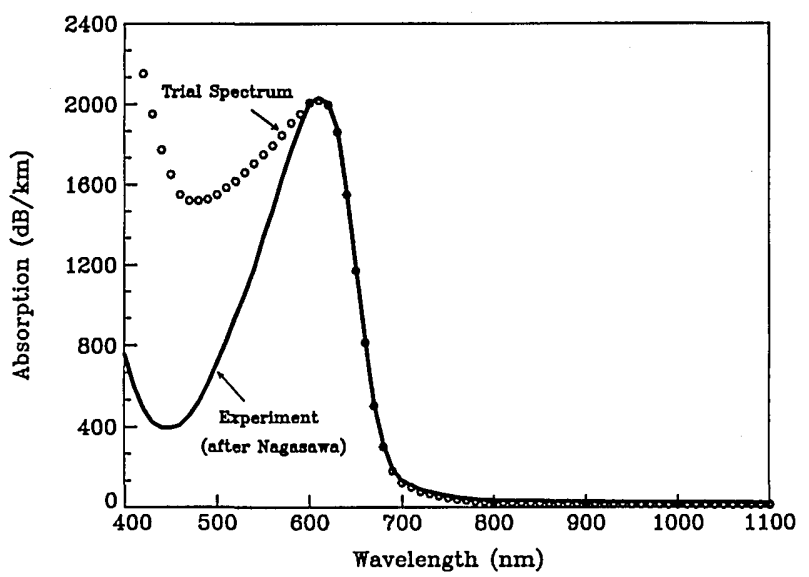

Fig. 4. Absorption spectrum of a $\gamma$-irradiated ( $12 \mathrm{kGy}$ ) pure-silicacore/F-doped-silica-clad fiber (solid curve) ${ }^{13}$ and trial spectrum (circles) used in model calculations of Fig. 5. 
are considered undesirable and are removed (mode stripped) by first removing the jacket from a segment of fiber near the input end and then conducting the bare fiber through a sinuous path immersed in indexmatching fluid. In the present experiment, mode stripping was not used during neutron irradiation. Therefore the possibility was considered that the observed artifact manifested in Fig. 3 can be related to the presence of copropagating cladding modes that respond differently to the neutron beam than do the core modes. This possibility was mathematically modeled by formally decomposing the transmitted light intensities in Eq. (1) in the following way:

$$
\begin{gathered}
I=I(\text { core })+I(\text { clad }), \\
I_{0}=I_{0}(\text { core })+I_{0}(\text { clad }),
\end{gathered}
$$

where $I$ (core) $=A \exp (-20 \alpha), I_{0}($ core $)=A \exp (-\alpha)$, $I($ clad $)=B \exp (-20 \beta)$, and $I_{0}($ clad $)=B \exp (-\beta)$. Here, $A$ and $B$ represent the intensities injected into the core and cladding, respectively, and $\alpha$ and $\beta$ are the respective absorption coefficients in inverse meters. The multipliers in the exponents are peculiar to the present experiment, where $I$ pertains to a $20-\mathrm{m}$ sample coil and $I_{0}$ pertains to a $1-\mathrm{m}$ reference coil, both of which are irradiated. For convenience, it was further assumed that the core absorption coefficient $\alpha$ takes the form $\alpha=\phi f(\lambda)$, where $\phi$ is the neutron fluence and $f(\lambda)$ is a suitably normalized spectral shape function consistent with known radiation-induced-absorption spectra for pure silica (e.g., the shapes in Fig. 4). Similarly, $\beta$ was taken proportional to the product of fluence and a reasonable spectral shape function $g(\lambda)$ for F-doped silica. The ratio $B /(A+B)$ represents the fraction of the launched light that is propagating in the cladding in the lead-in fibers. A key issue here is the fact that little is known about $g(\lambda)$ for F-doped silica; several possible forms were tried in the modeling but, in the end, the shape of the absorption induced in the cladding proved immaterial so long as $g(\lambda) \ll f(\lambda)$ for all $\lambda$.

In trial and error fashion, both $B /(A+B)$ and $f(\lambda)$ were varied and the calculated loss spectra $L[A, B$, $\phi f(\lambda)]$ were compared with experiment as a function of fluence $\phi$. The trial shape functions, $f(\lambda)$, were based on actual experimental data recorded by $\mathrm{Na}$ gasawa et al. for a $\gamma$-irradiated pure-silica-core/Fdoped-silica-clad fiber. ${ }^{13}$ Variations from the data of Nagasawa et al were constrained to major variations in the UV tail extending from below $400 \mathrm{~nm}$ to $\sim 600$ $\mathrm{nm}$, very minor changes in the nearly wavelengthindependent IR region (aside from removal of the $\mathrm{OH}$ bands), and no changes at all in the $630-\mathrm{nm}$ band. The most successful modeling achieved under these constraints involved an $f(\lambda)$ given by the circles in Fig. 4 and $B /(A+B) \approx 0.01$ [although varying $B$ / $(A+B)$ by a factor $\sim 10$ gave similar results $]$.

The results of the modeling are illustrated in Fig. 5. Even though no systematic optimization process was applied, Figs. 3 and 5 are qualitatively very similar. Moreover, above $\sim 680 \mathrm{~nm}$, the curves labeled 51 in

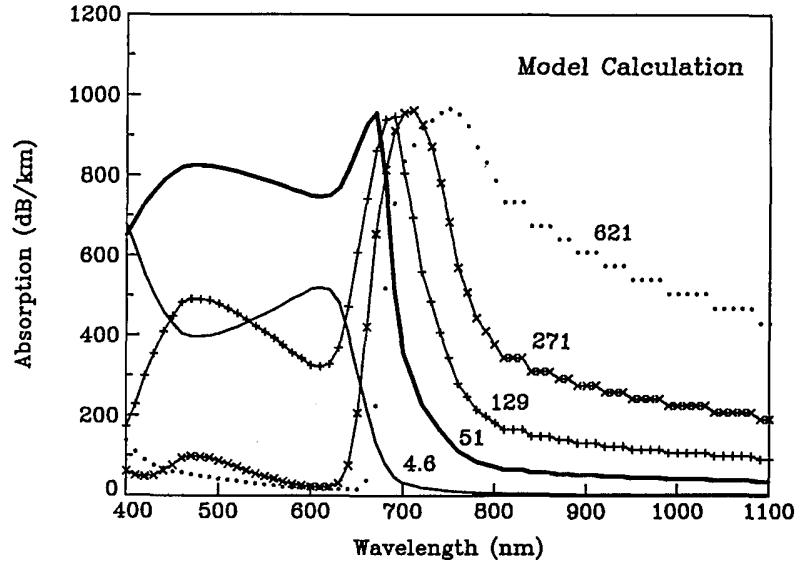

Fig. 5. Model calculation of radiation-induced absorption in a pure-silica-core optical fiber supporting low-level light transmission in a rad-hard optical cladding. The shape of the induced absorption in the core was assumed to be independent of fluence and was taken to be given by Fig. 4 (circles). The numbers on the curves are proportional to the intensities assumed for the core absorption. (These numbers could represent fluences if the induced losses were to grow linearly with fluence.)

Figs. 3 and 5 are essentially indentical. Discrepancies between other correspondingly labeled pairs of curves appear to be mostly ascribable to the limited validity of the model assumption that the absorption grows linearly with neutron dose $[\alpha=\phi f(\lambda)]$. Indeed, studies of pure-silica-core fibers under $\gamma$ irradiation typically exhibit near-linear growth in the dose range from 0 to $\sim 10^{2}$ Gy (where Gy is the absorbed dose in SI units) followed by a region of saturation (or slight bleaching) up to $\sim 10^{4} \mathrm{~Gy} .{ }^{2,3}$ Using the conversion $1 \mathrm{~Gy} \approx 3 \times 10^{11} \mathrm{n} / \mathrm{cm}^{2}$, the present neutron irradiations span a dose range of from $\sim 1.5 \times 10^{3}$ to $2 \times 10^{5} \mathrm{~Gy}$. Thus if the earlier $\gamma$-irradiation data are any indication, sublinear growth in $L(\lambda)$ is expected over most of the range of fluences represented in the present experiment. If this expectation were to be incorporated in the modeling of Fig. 5 , it is likely that better agreement with Fig. 3 could be achieved. However, the present results are sufficient to demonstrate that light travelling in the presumably rad-hard cladding of the CeramOptec fiber is almost certainly responsible for the clearly artifactual behavior evident in Fig. 3. A more general discussion of this cladding-mode hypothesis is given in Appendix A.

\section{Postirradiation Test}

To test the cladding-mode hypothesis further, an additional experiment was run approximately a month after the LAMPF beam was turned off. This was a classical cutback experiment performed on the 1-m coil of the CeramOptec fiber. (The 20-m coil was abandoned at that point because of its effective opacity below $\sim 700 \mathrm{~nm}$.) In this experiment only, $I$ was taken as the light through the 1-m coil and its associated lead-in and lead-out fibers, and $I_{0}$ was taken to be the light exiting from the lead-in fiber originally leading to the $20-\mathrm{m}$ coil but now cut back to 
within $1 \mathrm{~m}$ of the fiber-optic coupler. This cutback fiber was spliced directly to the spectrometer end of the corresponding lead-out fiber, which was itself cut within $1 \mathrm{~m}$ of the spectrometer. Two sets of data were obtained: one set before and one set after mode stripping the fibers near their entrance to the spectrometer. The optical losses determined from these results are shown in Fig. 6, where the effective length of irradiated fiber was arbitrarily taken to be $5 \mathrm{~m}(1-\mathrm{m}$ coil plus an assumed $4 \mathrm{~m}$ to account for the nonuniformly irradiated pigtails). Below $\sim 650 \mathrm{~nm}$, a factorof- $\sim 2$ attenuation is noted in the mode-stripped case vis-à-vis the measurement without mode stripping. This is qualitatively the kind of effect expected if a sufficient proportion of the light were to be light guiding in the relatively rad-hard claddings and thus bypassing the radiation-darkened portions of the fiber cores.

An effort was made to measure directly the amount of light launched into cladding modes by taking the difference between the values of $I_{0}$ (the light exiting from the cutback length) recorded before and after mode stripping. Because of the necessity of physically moving the bare fibers in this process, the reproducibility of these measurements was assumed to have been no better than $\sim 1 \%$. Nevertheless, measurements at 600 and $700 \mathrm{~nm}$ consistently indicated $\sim 1 \%$ of the light to be traveling in the cladding at the injection end (i.e., within $\sim 1 \mathrm{~m}$ of the fiberoptic coupler). In the case of the 1-m irradiated sample coil, $I(\lambda)$ measured before and after stripping showed that $\sim 50 \%$ of the exiting light at $700 \mathrm{~nm}$ and $\sim 95 \%$ of the exiting light at $600 \mathrm{~nm}$ was by that poinit traveling in the cladding. These observations are again qualitatively consistent with the assumed mode distribution at the injection end $[B /(A+B) \approx$ $0.01]$ used in the model calculations. However, at $600 \mathrm{~nm}$ the absolute light intensity propagating in

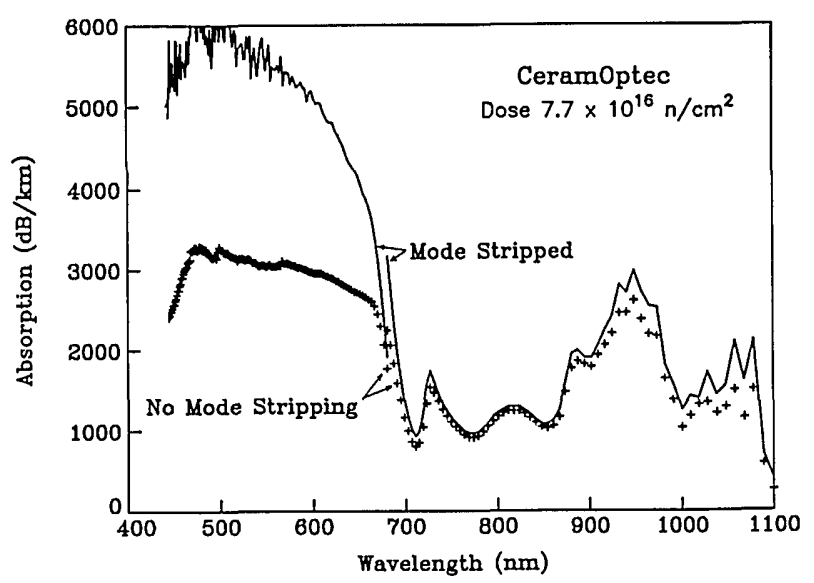

Fig. 6. Postirradiation measurement of absorption spectrum of CeramOptec fiber (1-m coil in beam, plus lead-in and lead-out fibers) obtained by cutback technique. Below $\sim 700 \mathrm{~nm}$, the spectrum obtained with mode stripping gives the true spectral shape of the optical loss in the fiber core, although its absolute value is uncertain within a factor of $\sim 2$. Above $700 \mathrm{~nm}$, the induced loss in coil is not separable from the intrinsic loss in leads. the cladding at the exit end of the $1-\mathrm{m}$ coil was found to be $\sim 5$ times greater than the light intensity propagating in cladding modes in the cutback length (reproducibility problems hindered measurements at longer wavelengths). Thus the possibility remains that significant light may have leaked from core to cladding modes along the fiber lengths closer to, or in, the neutron beam. One possible mechanism of leaking core modes into the cladding is suggested by the results of optical time-domain reflectometer measurements, which showed a high degree of light backscattering in the irradiated regions of the sample coils, this scattering building up concomitantly with the induced absorption. ${ }^{16}$

\section{Discussion}

It can be concluded from the above measurements and modeling that fast-neutron irradiation induces substantially higher optical losses in the pure-silica core of the CeramOptec fiber than in the F-dopedsilica cladding. This situation, in connection with the demonstrated fact that cladding modes were being propagated, explains the artifact of Fig. 3, which was in turn simulated in Fig. 5. Although such an artifact does not appear to have been reported previously, there are some precedents in the literature that shed additional light on the phenomenon.

Recently, West and Dowling ${ }^{17}$ studied the effects of $\gamma$ irradiation on certain fibers propagating cladding modes. These authors anticipated that "Any light propagating in the cladding would be expected to show greater transmission loss than lower-order guided modes both normally and particularly under the effects of irradiation." This expectation was confirmed at $850 \mathrm{~nm}$ by means of optical time-domain reflectometer studies of a fiber composed of Ge:Pdoped silica. In the present context, it is necessary to remark that Ge:P-type fibers typically contain phosphorus in the cladding and that such phosphorusdoping is known to lead to $\gamma$-ray-induced optical attenuation that is typically 2 orders of magnitude higher than for pure silica. ${ }^{18}$ Quite a different effect might have been anticipated for an F-doped cladding material, because neutron and $\gamma$ irradiation effects in F-doped-silica-core fibers (at wavelengths 820-850 $\mathrm{nm}$ ) have been found to be quite comparable to those in pure-silica-core fibers. ${ }^{8}$

Particularly germane to understanding the results of the present experiment are the results of $\mathrm{Na}$ gasawa et al. ${ }^{19}$ concerning the effects of cladding material on the radiation-induced $2-\mathrm{eV}(630-\mathrm{nm})$ optical absorption in pure-silica-core fibers. These authors examined pure-silica-core fibers of differing $\mathrm{OH}$ contents and differing manufacturing processes that were provided with optical claddings of either silicone polymer or F-doped silica. In every instance the $2-\mathrm{eV}$ band strength per unit $\gamma$-ray dose was approximately an order of magnitude higher in the F-doped-silica-clad fiber's than in the polymer-clad counterparts. However, this result was shown to be 
because of losses induced in the pure-silica cores and not the F-doped silica claddings. In an earlier paper,,$^{12}$ Nagasawa et al. successfully correlated the $2-\mathrm{eV}$ absorption band with the electron-spin-resonance spectrum of the nonbridging-oxygen hole center (see Ref. 20 for a recent review of this subject). Using this knowledge, Nagasawa et al. ${ }^{19}$ were able to determine by an etchback method the radial distribution of nonbridging-oxygen hole centers (i.e., the $2-\mathrm{eV}$ defect center) in their fibers. They found that the $2-\mathrm{eV}$ defect was approximately an order of magnitude more abundant in the core near the core/cladding interface than in the F-doped cladding. These near-interface defects were absent in the silicone-clad fibers. The results of Nagasawa and coworkers' radial profiling experiment are fully consistent with the interference that the 2-eV band in the CeramOptec fiber of the present study is at least an order of magnitude more intense in the core than in the cladding.

Finally, it must be remarked that Nagasawa et al. did not observe any hint of an artifact of the nature of the one illustrated in Figs. 3 and 5. This is in spite of the fact that Nagasawa did not mode strip and, in fact, used a mode scrambler near the launch end, ${ }^{21}$ which would in principle have filled the cladding modes. It is necessary to assume therefore that Nagasawa's glass-clad fibers were jacketed with a higher-index polymer or a polymer that was considerably more lossy than the polymer on the CeramOptec fiber of the present investigation. Nor was this artifact observed during concurrent experiments at LASREF with a different suite of fibers and a diodearray spectrophotometer. ${ }^{16}$ The reason again may be related to lossy polymer jacketings, although the use of fiber-optic couplers, rather than fusion splices, could also have had a negative effect on the throughput of any cladding modes.

\section{Conclusions}

The present results are explained by the existence of the following set of conditions in the fibers investigated:

1. Both the sample and reference fibers are being irradiated;

2. the induced absorption in the core eventually becomes much greater than that in the optical cladding;

3. the intrinsic (as well as induced) loss in the protective polymer jacket is not too great;

4. a sufficiently large fraction of the injected light is leaking into cladding modes; and

5. no mode stripping is being used on the fiber leads returning to the spectrometer.

That the kind of artifact represented by Fig. 3 has not been reported before, to our knowledge, is probably because all five of these conditions have not been simultaneously satisfied previously. Condition 1 held several advantages in the current context (CCDcamera detection and highly remote sample coils with the pigtails also subject to irradiation), whereas most $\gamma$-irradiation experiments in the literature have not required this arrangement. Still there is independent evidence that condition 2 is common for puresilica-core/F-doped-silica-clad fibers ${ }^{19}$ as well as for Ge-doped-silica-core/pure-silica-clad fibers. ${ }^{2-4,8}$ Thus even without condition 1 , effects such as are revealed in Fig. 6 might have been seen if conditions 3 through 5 had been satisfied. Condition 3 depends strongly on the cladding material, for which many possibilities exist. Perhaps the unique situation in the present experiment was the LASREF neutron beam itself, which evidence suggests may be responsible for condition 4 by creating numerous recoil ions whose tracks scatter light from the core to the cladding. Condition 5 is a relatively common practice, because the standard protocols for measuring radiation-induced attenuation in optical fibers call for optional mode stripping only at the injection ends. ${ }^{22}$

The inferred rad hardness of the F-doped-silica cladding material, coupled with Nagasawa's conclu$\operatorname{sion}^{20}$ that most absorption of visible light is associated with manufacturing-induced near-interface precursor defects in the pure $\mathrm{SiO}_{2}$ core, suggests a possible structure for a relatively rad-hard fiber for fusion reactor diagnostic applications. This structure would be a graded-index (interface-free) fiber based entirely on F-doped silica. Indeed, Schneider and $\mathrm{Babst}^{8}$ found such fibers to perform quite well at $\lambda=820-850 \mathrm{~nm}$ under neutron irradiation. Full spectral studies of fibers having this construction are planned for future investigations:

\section{Appendix A}

The artifactual behavior apparent in the experimental data of Fig. 3 and the mathematical modeling of Fig. 5 can be understood intuitively as follows: Assuming that significant light intensities are propagating in both the cores and claddings of both the $20-\mathrm{m}$ sample and 1-m reference lengths of fiber and that the claddings are relatively rad hard, three successive stages are to be expected in the optical losses determined by Eq. (1) as a function of increasing radiation damage. In Stage $I$, the amount of light intensity propagating in the cladding is relatively trivial, so the initial absorption coefficient of the fiber is essentially that of the core, i.e., $\alpha(\lambda)$. For this reason, in Fig. 5 the curve labeled 4.6 turns out to be virtually identical in shape with $\alpha(\lambda)=\phi f(\lambda)$, where $f(\lambda)$ is the trial spectrum of Fig. 4 (circles). In Stage II, the optical density of the core of the sample fiber, $20 \alpha(\lambda)$, becomes so great that the light propagating in the cladding is found to be comparable to or greater than the light in the core in the wavelength range below $\sim 650 \mathrm{~nm}$. At this stage, the apparent loss in the sample fiber saturates in the latter wavelength range. Further increases in dose do not alter this situation in the sample fiber, but they do begin to bring on the same situation in the reference fiber. This means that in Stage II the light through the reference fiber in the range below $\sim 650$ 
$\mathrm{nm}$ begins to drop, whereas the light through the sample fiber remains relatively constant. Thus the result of calculating an optical density $\operatorname{OD}(\lambda)=$ $\log \left(I_{\text {ref }} / I_{\text {sample }}\right)$ shows a negative absorption, proportional to $-\alpha(\lambda)$, growing in this dose range. This effect is seen clearly in Fig. 5, where in the range $\lambda<$ $650 \mathrm{~nm}$ the curve labeled 129 is virtually identical to an inverted curve 4.6. Finally, in Stage III, the core regions of both the sample and reference coils become effectively opaque relative to the claddings. From this point on the optical loss calculated with Eq. (1) becomes the loss in the cladding alone. Thus the dotted curve (621) in Fig. 5 for the wavelength range below $\sim 650 \mathrm{~nm}$ turns out to be identical with the shape $g(\lambda)$ that is assumed in the calculation for the induced loss in the F-doped cladding material. It is emphasized that the progression from Stage I to Stage III has a spectral dependence as well as a dependence on fluence. Thus in Fig. 5, Stage III is reached only at the higher fluence and only below $\sim 650 \mathrm{~nm}$ with the assumed $g(\lambda)$. Between $\sim 650$ and $\sim 800 \mathrm{~nm}$, an evolution from Stage I to Stage II is seen as a function of increasing fluence. Above $\sim 800 \mathrm{~nm}$, the transmitted light remains predominately in guided core modes for all fluences considered, and the calculated curves do not progress beyond Stage I.

The authors thank E. H. Farnum and W. Sommer (Los Alamos National Laboratory) for their hospitality and assistance at Los Alamos Spallation Radiation Effects Facility and for the provision of dosimetry and temperature data. Discussions with C. Askins (Naval Research Laboratory) contributed materially to the design of the experiment. This work was supported by the U.S. Department of Energy Office of Energy Research.

\section{References and Notes}

1. F. W. Clinard, Jr., E. H. Farnum, D. L. Griscom, R. F. Mattas, S. S. Medley, F. W. Wiffin, S. S. Wojtowwicz, K. M. Young, and S. J. Zinkle, "Materials issues in diagnostic systems for BPX and ITER,” J. Nucl. Mater. 191-194, 1399-1403 (1992).

2. E. J. Friebele, "Optical fiber waveguides in radiation environments,” Opt. Eng. 18, 552-561 (1979).

3. E. J. Friebele, M. E. Gingerich, and K. J. Long, "Radiation damage of optical fiber waveguides at long wavelengths," Appl. Opt. 21, 547-553 (1982).

4. E. J. Friebele, C. G. Askins, M. E. Gingerich, and K. J. Long, "Optical fiber waveguides in radiation environments, II," Nucl. Instrum. Methods B 1, 355-369 (1984).

5. E. J. Friebele, K. J. Long, C. G. Askins, M. E. Gingerich, M. J. Marrone, and D. L. Griscom, "Overview of radiation effects in fiber optics," in Effects on Optical Materials, P. W. Levy, ed., Proc. Soc. Photo-Opt. Instrum. Eng. 541, 70-88 (1985).

6. P. B. Lyons, "Fiber optics in transient radiation fields," in Radiation Effects on Optical Materials, P. W. Levy, ed., Proc. Soc. Photo-Opt. Instrum. Eng. 541, 89-96 (1985).
7. J. K. Partin, "Fiber optics in high dose radiation fields," in Radiation Effects on Optical Materials, P. W. Levy, ed., Proc. Soc. Photo-Opt. Instrum. Eng. 541, 97-109 (1985).

8. W. Schneider and U. Babst, "Induced attenuation in optical fibers during steady state and pulsed irradiation," presented at Photon '83, 1983.

9. Neutron fluences quoted here are preliminary estimates provided by W. Sommer of Los Alamos National Laboratory based on earlier determinations of fluence as a function of integrated beam current at LASREF. This estimate could be in error by as much as a factor of 2 , because of the as-yet unevaluated effects of isotope production stringers having been inserted into the beam at various times during the current run.

10. D. B. Keck, R. D. Maurer, and P. C. Schultz, "On the ultimate lower limit of attenuation in glass optical waveguides," Appl. Phys. Lett. 22, 307-309 (1973).

11. E. J. Friebele and M. E. Gingerich, "Radiation-induced optical absorption bands in low loss optical fiber waveguides," J. Non-Cryst. Solids 38/39, 245-250 (1980).

12. K. Nagasawa, M. Tanabe, and K. Yahagi, "Gamma-ray induced absorption bands in pure silica core fibers," Jpn. J. Appl. Phys. 23, 1608-1613 (1984).

13. K. Nagasawa, Y. Hoshi, Y. Ohki, and K. Yahagi, "Radiation effects on pure silica core optical fibers by $\gamma$-rays: relation between $2 \mathrm{eV}$ band and non-bridging oxygen hole center," Jpn. J. Appl. Phys. 25, 464-468 (1986).

14. S. E. Miller and A. G. Chynoweth, Optical Fiber Telecommunications (Academic, San Diego, Calif., 1979).

15. S. E. Miller and I. P. Kaminow, Optical Fiber Telecommunications II (Academic, San Diego, Calif., 1988).

16. Optical time-domain reflectometer data taken by W. Unruh will be published [W. Unruh, "In situ measurements of optical absorption due to high-flux neutron irradiation of silica optical fibers," to be submitted to Appl. Opt.].

17. R. H. West and S. Dowling, "Measurement of long term radiation induced losses in fibre optics using optical time domain reflectometry," in Proceedings of the First European Conference on Radiation and Its Effects on Devices and Systems (RADECS 91) (Institute of Electrical and Electronics Engineers, New York, 1992), pp. 375-379.

18. D. L. Griscom, E. J. Friebele, K. J. Long, and J. W. Fleming, "Fundamental defect centers in glass: electron spin resonance and optical absorption studies of irradiated phosphorusdoped silica glass and optical fibers," J. Appl. Phys. 54, 3743-3762 (1983).

19. K. Nagasawa, R. Tohmon, and Y. Ohki, "Effect of cladding material on $2 \mathrm{eV}$ optical absorption in pure-silica core fibers and method to suppress the absorption," Jpn. J. Appl. Phys. 26, 148-151 (1987).

20. D. L. Griscom, "Optical properties and structure of defects in silica glass," J. Ceram. Soc. Jpn. 99, 923-942 (1991).

21. K. Nagasawa, Shonan Institute of Technology, Fujisawa, Kanagawa 251, Japan (personal communication, 1992).

22. E. J. Friebele, chairman, and NATO Nuclear Effects Task Group A/C243, Panel IV (RSG.12), "Procedure for measuring radiation-induced attenuation in optical fibers and optical cables," Rep. NRL/MR/6505-92-6963 (Naval Research Laboratory, Washington, D.C., 1992), pp. 1-21. 